\title{
Análisis de los factores de radiación-evapotranspiración en el Desierto Sonorense
}

\author{
Analysis of radiation-evapotranspiration factors in the Sonoran Desert
}

\author{
Mayra Guadalupe Gaxiola-Morales*, Luis Brito-Castillo \\ Centro de Investigaciones Biológicas del Noroeste S.C., Campus Guaymas. Km. 2.35 Camino al Tular Estero de Bacochibampo \\ CP 349, Heroica Guaymas, Sonora, México.
}

\section{RESUMEN}

En este trabajo se identificaron las épocas del año en que la pendiente de regresión entre datos calibrados de albedo y de temperatura cambia de signo. Si la relación es decreciente, indica que el factor que domina la modulación de la temperatura superficial es el de radiación. Por el contrario, si la relación es creciente, el factor que domina la modulación de la temperatura superficial es el de evapotranspiración. El cambio en la dominancia de un factor respecto al otro en la modulación de la temperatura puede servir de base para entender cuando las condiciones húmedas de un lugar, donde domina el factor de evapotranspiración, llegan a un estado de deshidratación que afecta a las plantas dando paso a condiciones típicas de desierto, en las que domina el factor de radiación. El estudio se realizó en diferentes sectores del Desierto Sonorense, en un gradiente latitudinal sur-norte con diferencias en tipos de suelo y vegetación. Los resultados indican cambios significativos en las relaciones de albedo vs. temperatura, asociados a las diferencias locales en cada sector; al considerar la vegetación y suelo, en los meses con precipitación domina el factor de evapotranspiración y en temporada seca, el factor dominante es de radiación.

Palabras clave: Radiación, Evapotranspiración, Desierto Sonorense

\section{ABSTRACT}

In this study, the seasons when the slop of the regression line between albedo and surface temperature changes the sign are identified. A decreasing relationship between both characteristics indicates that the surface temperature modulating factor is radiation. On the contrary, an increasing relationship between both characteristics indicates that the surface temperature modulating factor is evapotranspiration. The change, being indicative of the reverse in the dominance of one factor relative to the other in modulating surface temperature, can be used to comprehend when moisture conditions of one place, where evapotranspiration factor dominates, desiccate affecting the vegetation and producing desert-like conditions, where radiation factor dominates. The study was done in different sectors of the Sonoran Desert, through a south-north latitude gradient with differences in soil types and vegetation. The results indicate significant changes in the relationships of albedo vs. temperature, associated with local differences in each sector; considering vegetation and soil, in the precipitation months the evapotranspiration factor dominates while in the dry season, the dominant factor is radiation.

Keywords: Radiation, Evapotranspiration, Sonoran Desert

\section{INTRODUCCIÓN}

La cantidad de energía que ingresa a la Tierra está determinada, en gran medida por la constante solar y el albedo (Pallé et al., 2008). Se define albedo como la fracción de la energía solar incidente a la Tierra que es regresada al espacio por reflexión o dispersión, sin ser absorbida y sin cambios en sus longitudes de onda (Taylor, 2005); es de suma importancia debido a su participación en la regulación de la temperatura superficial.

Se han realizado esfuerzos por estudiar los mecanismos de regulación de la temperatura en la Tierra; algunos autores como Otterman (1974) y Charney (1975), plantearon lo que se conoce como la hipótesis del albedo, con el fin de explicar las diferencias de temperaturas observadas entre la península del Sinaí, Egipto, zona que vista desde el espacio muestra superficies claras, y el noroeste de Negev, con superficies oscuras y vegetación degradada. Los autores realizaron mediciones a finales de agosto de 1973, y mostraron una diferencia de $5^{\circ} \mathrm{C}$ entre estas dos regiones $\left(40^{\circ} \mathrm{C}\right.$ en la península del Sinaí y $45^{\circ} \mathrm{C}$ en el Desierto del Negev). Posteriormente, repitieron las mediciones a finales de febrero de 1974 , mostrando una diferencia de $3.5^{\circ} \mathrm{C}\left(30^{\circ} \mathrm{C}\right.$ en el lado claro y $33.5^{\circ} \mathrm{C}$ en el lado oscuro). Estos resultados permitieron sugerir que entre mayor es el albedo de un sitio menor es su temperatura superficial, es decir, hay una relación inversa entre el albedo y la temperatura, ya que al incrementarse el albedo la radiación de onda corta absorbida por la superficie disminuye, provocando enfriamiento de la superficie. Esta relación está dominada por el factor de radiación (Tereshchenko et al., 2012).

En otros estudios se verificó que la hipótesis del albedo solo es válida en los desiertos (Zolotokrylin, 2003), con valores muy altos de albedo y poca vegetación, que son características típicas de los desiertos Sonorense y Chihuahuense; por lo tanto, se ha sugerido al menos existe otro factor que modula la temperatura superficial, el factor de evapotranspiración (Tereshchenko et al., 2012). El factor de evapotranspiración tiene que ver con los componentes de balance de energía a nivel local. Al reducirse la vegetación sea por falta de precipi-

Volumen XXI, Número 2 
tación o por acción del hombre se incrementa el albedo, ya que la superficie se vuelve más clara. También disminuye la evapotranspiración y se calienta la superficie debido a que el calor que era utilizado por las plantas para transpirar ya no se utiliza más, ya que hay menos vegetación, y en consecuencia el calor pasa directamente hacia la superficie calentándola.

El efecto contrario también ocurre, es decir, al incrementarse la precipitación hay un incremento de la vegetación y la superficie es más opaca, se absorbe más radiación y disminuye el albedo, reduciendo el calentamiento de la superficie (Vivoni et al., 2008).

Por lo tanto, el factor de evapotranspiración se distingue como una relación directa albedo-temperatura superficial.

En algunos sectores del Desierto Sonorense con zonas claras, muy poca o nula vegetación y valores altos de albedo (entre 20-35\%, como el Desierto de Altar), es factible afirmar que el factor que domina la modulación de la temperatura es el de radiación; mientras que en zonas donde se incrementa la humedad, la vegetación aumenta y los valores de albedo disminuyen (es decir, son $<20 \%$ ), es factible afirmar que el factor que domina la modulación de la temperatura es el de evapotranspiración. El objetivo de este trabajo fue verificar si estas afirmaciones son correctas. Estos resultados son útiles para identificar la posible existencia de un valor umbral que sea indicador del cambio en la dominancia de un factor (por ejemplo el de radiación, que es típico de condiciones de desierto), respecto del otro (el de evapotranspiración, que es típico de presencia de vegetación y humedad del suelo). Más aún, los resultados pueden ser útiles para identificar el inicio de una sequía.

\section{MATERIALES Y MÉTODOS}

Para lograr el objetivo propuesto, se analizaron las correlaciones entre el albedo y la temperatura superficial en diferentes sectores del Desierto Sonorense. Se identificaron las épocas del año en que el signo de la pendiente cambia y se evaluó la significancia estadística de las correlaciones. Se verificó además si los cambios en las correlaciones entre el albedo y la temperatura superficial fueron consistentes con los observados en las variaciones de las precipitaciones en un gradiente latitudinal. Se discutieron estas relaciones para determinar la alternancia entre los dos factores que modulan la temperatura del aire.

\section{Área de estudio}

El Desierto Sonorense se ubica en la región árida del noroeste de México entre $23^{\circ}-35^{\circ} \mathrm{N}$ y $109^{\circ}-117^{\circ} \mathrm{O}$, cubriendo una extensión de $260,000 \mathrm{~km}^{2}$, en la mitad sur de Arizona y sureste de California en Estados Unidos, la mayor parte de la Península de Baja California, algunas islas del Golfo de California y una gran proporción del estado de Sonora (Figura 1). Comparado con los otros tres desiertos que existen en Norte América (El Chihuahuense, el de La Gran Cuenca y el de Mojave), el Desierto Sonorense es el más septentrional, el más húmedo y cálido de los cuatro y probablemente el desierto

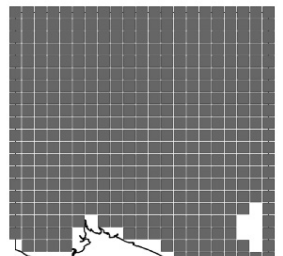

C5 $=409$ pixeles

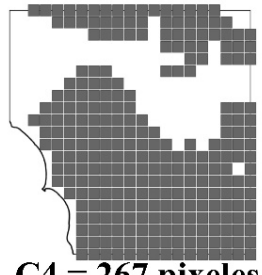

C4 $=267$ pixeles
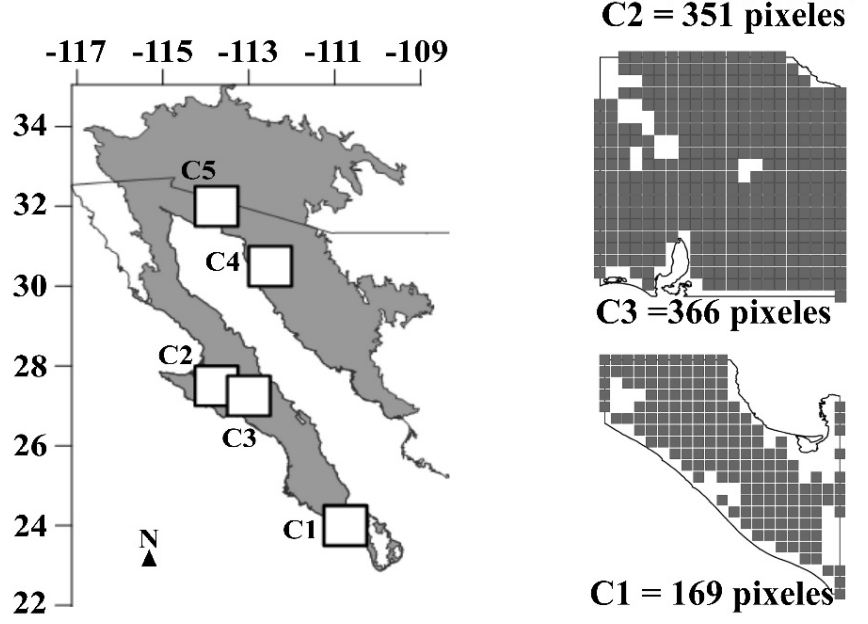

Figura 1. Ubicación del área de estudio. El área sombreada indica los límites del Desierto Sonorense (Shreve y Wiggins, 1964). C1-C5 son los cuadrantes seleccionados. Fuera del mapa, las zonas oscuras muestran el número de pixeles filtrados en cada cuadrante una vez removidos los pixeles localizados en zonas agrícolas y urbanas.

Figure 1. Location of study area. The shaded area indicates the borders of the Sonoran Desert (Shreve y Wiggins, 1964). C1-C5 are the selected squares. Dark areas outside the map display the number of filtered pixels, once the pixels located in agricultural and urban areas were removed.

botánicamente más rico del mundo ya que soporta una rica variedad de flora y fauna (Shreve y Wiggins, 1964).

En la porción central de la península de Baja California se encuentra El Vizcaíno, en Baja California Sur, un área extensa de desierto entre dos mares, el Golfo de California y el Océano Pacífico, con abundantes dunas de arena, vegetación halófita, lagunas costeras y manglares (INEGI, 2013a). En la porción noroccidental de Sonora se localiza el Desierto de Altar que cubre también la región del Pinacate, una de las regiones más áridas del mundo donde la precipitación anual es entre 50 y $100 \mathrm{~mm}$. La precipitación anual en el Desierto Sonorense se encuentra entre 100 y 300 mm; se distribuye de manera irregular a lo largo del año incrementándose paulatinamente hacia el sur (Figura 2) debido a la mayor contribución de los sistemas tropicales. Ocurre principalmente en verano entre julio y septiembre cuando las temperaturas superan los $40^{\circ} \mathrm{C}$ a la sombra y está controlada por el monzón de Norteamérica (Douglas et al., 1993; Higgins et al., 2003). Hay otro período de lluvia entre noviembre y marzo, cuya contribución es entre 30 y $40 \%$ en relación con el total anual y se asocia al paso de los sistemas de latitudes medias y sus frentes extratropicales (Jáuregui, 1995). Los períodos secos se observan a principios de verano (Mayo-Junio) y a principios de otoño. 


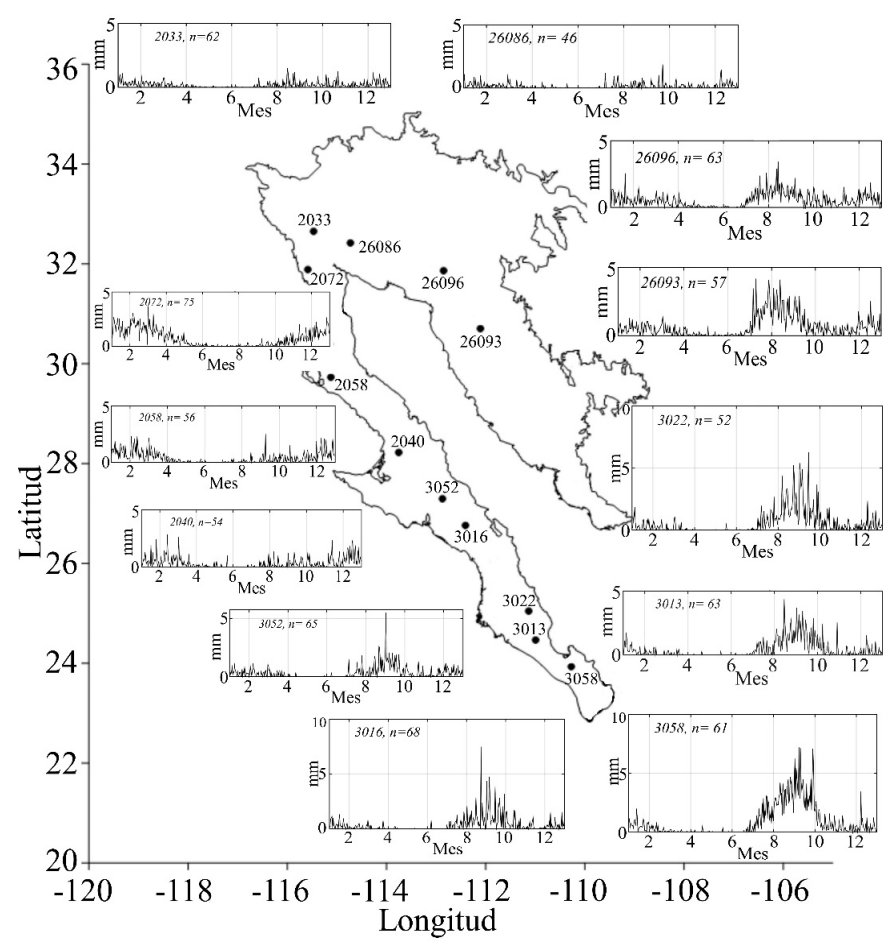

Figura 2. Climatología diaria de precipitación de algunas estaciones localizadas en el Desierto Sonorense y que contienen registros con más de 40 años. Se muestra en cada caso la clave de la estación y el número de años con que se realizó el cálculo.

Figure 2. Daily precipitation climatology based on over 40-year records, derived from some weather stations located in the Sonoran Desert. In each case, the station code and the numbers of years of calculation is shown.

Se seleccionaron cinco cuadrantes de $1 \times 1^{\circ}$ (aproximadamente 100 kilómetros lineales en una matriz de $21 \times 21$ pixeles de $5.6 \mathrm{~km}$, Figura 1) en un gradiente latitudinal con diferencias en vegetación, características de suelo y patrón de distribución de lluvias (Figura 2). Los tres primeros cuadrantes se ubican en la península de Baja California, el primero (C1) en el municipio de La Paz (entre las coordenadas $23^{\circ} 30^{\prime}-24^{\circ} 30^{\prime} \mathrm{N}$ y $\left.110^{\circ} 15^{\prime}-111^{\circ} 15^{\prime} \mathrm{O}\right)$, el segundo ( $\mathrm{C} 2$, coordenadas $27^{\circ}-28^{\circ} \mathrm{N}$ y $113^{\circ} 15^{\prime}-114^{\circ} 15^{\prime} \mathrm{O}$ ) y tercero (C3, coordenadas $26^{\circ} 45^{\prime}-27^{\circ} 45^{\prime} \mathrm{N}$ y $112^{\circ} 30^{\prime}-113^{\circ} 30^{\prime} \mathrm{O}$ ), dentro de la Reserva de la Biosfera El Vizcaíno. El cuarto cuadrante (C4, coordenadas $30^{\circ}-31^{\circ} \mathrm{N}$ y $\left.112^{\circ}-113^{\circ} \mathrm{O}\right)$, en la porción media del estado de Sonora y el último cuadrante (C5, coordenadas $31^{\circ} 30^{\prime}-32^{\circ} 30^{\prime} \mathrm{N}$ y $113^{\circ} 15^{\prime}-114^{\circ} 15^{\prime} \mathrm{O}$ ) en la Reserva de la Biosfera El Pinacate.

De acuerdo a la Carta de Uso de Suelo y Vegetación (INEGI, 2013b) y la Carta de Edafología (INIFAP-CONABIO, 1995), C1 es un escenario compuesto por matorral sarcocaule y sarcocrasicaule, con suelos rocosos típicos de montaña y con tonalidad clara (Regosoles, Xerosoles y Yermosoles). C2 y C3 están compuestos por vegetación de matorral y vegetación halófila en su mayoría, con suelos claros, rocosos, enriquecidos con sales y secos (Regosoles, Solonchak's, Yermosoles). C4 y C5 están compuestos por vegetación de matorral desértico típica de desiertos arenosos, con suelos claros y rocosos (Fluvisoles, Litosoles, Regosoles). C5 cuenta con presencia de roca volcánica. En la Figura 3, se muestran los rangos promedio de albedo para diferentes condiciones de suelo que sirven de referencia a este trabajo.

\section{Preparación y procesamiento de datos}

Se utilizaron datos mensuales de albedo y temperatura superficial de imágenes de satélite distribuidos por el U.S. Geological Survey/National Aeronautics and Space Administration Land Processes Distributed Active Archive Center (LP DAAC; ver https://lpdacc.usgs.gov/) de los productos MCD43C3-V6 (Schaaf et al., 2015) y MODIS MOD11C3-V6 (Wan et al., 2015), para el periodo feb/2000-feb/2018, con resolución espacial de $5.6 \mathrm{~km}$. Los valores de albedo inferiores a $15 \%$ fueron removidos del análisis ya que no corresponde a ninguna de las condiciones descritas en la Figura 3.

Los pixeles localizados en mar, zonas agrícolas y zonas urbanas fueron removidos del análisis para evitar sesgos debido a influencia humana, quedando 169, 351, 366, 267 y 409 pixeles para los cuadrantes $\mathrm{C} 1-\mathrm{C} 5$, respectivamente (Figura 1).

Una vez removidos los pixeles mencionados, el análisis de regresión entre albedo y temperatura se realizó 1) tomando en cuenta todos los datos históricos disponibles y 2) tomando en cuenta los promedios históricos para cada mes. En cada caso se evaluó la significancia estadística y el signo de la pendiente. En este análisis se considera verano los meses de mayo-octubre, e invierno de noviembre-abril.

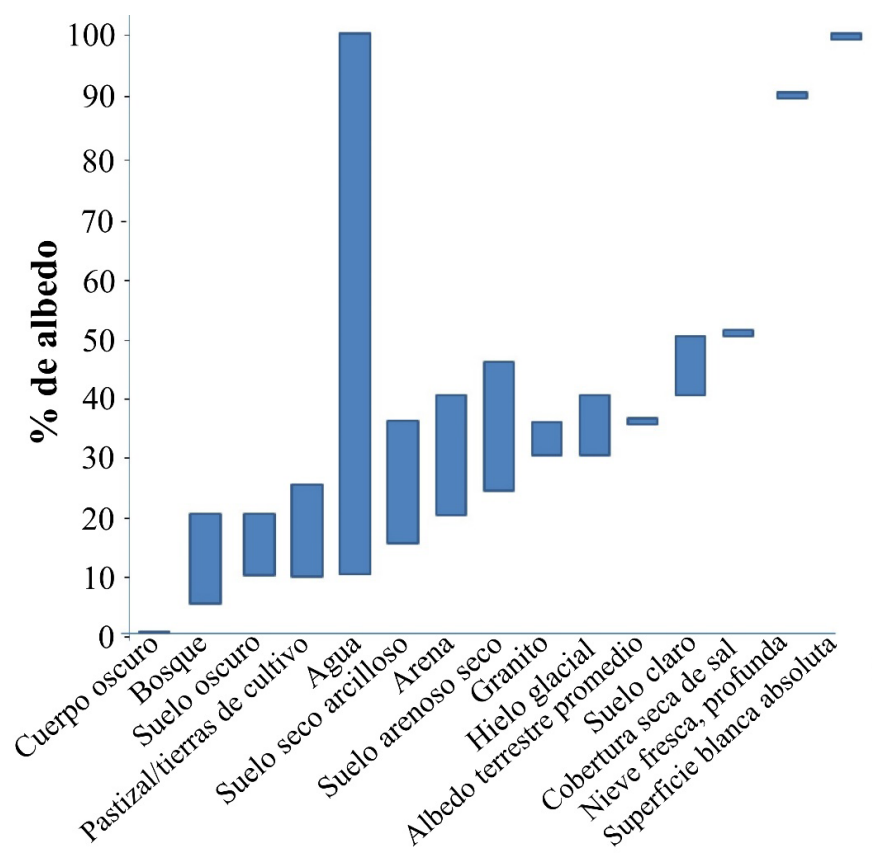

Figura 3. Rangos aproximados de albedo de superficies naturales. Fuente: Dobos, 2003.

Figure 3. The approximated ranges of albedo from natural surfaces. Source: Dobos, 2003. 


\section{Correlación albedo-temperatura por tipo de vegetación y tipo de suelo}

En los cuadrantes $\mathrm{C} 1-\mathrm{C} 5$ se tomó en cuenta la distribución de los diferentes tipos de vegetación y de suelo, según las cartas de Uso de Suelo y Vegetación (1:250 000) y la Carta de Edafología (1:250 000), con el fin de filtrar los pixeles localizados en cada tipo. En estos casos, el análisis de regresión solo se llevó a cabo cuando el número de pixeles en cada tipo fue $\geq 15$. En la Tabla 1 se muestra el número de pixeles identificados en cada polígono por tipo de vegetación y suelo.

Tabla 1. Número de pixeles identificados en C1-C5 por tipo de vegetación y de suelo.

Table 1. Number of pixels identified in C1-C5 by vegetation and soil type.

\begin{tabular}{|c|c|c|c|c|c|}
\hline Tipo & C1 & C2 & C3 & C4 & C5 \\
\hline Desierto arenoso & & 94 & 27 & 30 & 119 \\
\hline Encino & & & 2 & & \\
\hline Galería & 4 & 1 & 17 & 3 & \\
\hline Halófila & & 89 & 51 & 2 & 5 \\
\hline Matorral crasicaule & & & & & 1 \\
\hline Matorral desértico & & 52 & 20 & 144 & \\
\hline Matorral desértico micrófilo & 4 & & & & 109 \\
\hline Matorral sarcocrasicaule & 62 & 17 & 49 & & \\
\hline $\begin{array}{l}\text { Matorral sarcocrasicaule de } \\
\text { neblina }\end{array}$ & 10 & & & & \\
\hline Matorral sarcocaule & 116 & 82 & 203 & 72 & 25 \\
\hline Mezquital & 12 & & 2 & 10 & \\
\hline No aplicable & & 4 & 2 & 3 & \\
\hline Sin vegetación aparente & & 13 & 12 & & 27 \\
\hline Feozem háplico & & 12 & 13 & & \\
\hline Fluvisol calcárico & & 3 & & 19 & 28 \\
\hline Fluvisol éutrico & & 10 & 32 & 4 & \\
\hline Litosol & 3 & 28 & 180 & 47 & 38 \\
\hline Regosol calcárico & 25 & 66 & 25 & 55 & 171 \\
\hline Regosol éutrico & 51 & 149 & 152 & 58 & 10 \\
\hline Solonchak gléyico & & 14 & 9 & & \\
\hline Solonchak órtico & & 2 & 1 & & 4 \\
\hline Vertisol crómico & & 2 & 19 & 2 & \\
\hline Xerosol calcárico & & 5 & & 2 & \\
\hline Xerosol háplico & 37 & 5 & & 7 & \\
\hline Xerosol lúvico & & 1 & & 3 & \\
\hline Yermosol calcárico & 8 & 2 & & 17 & 26 \\
\hline Yermosol háplico & 39 & 19 & 28 & 53 & 10 \\
\hline Yermosol lúvico & 6 & 7 & & 1 & \\
\hline
\end{tabular}

El área sombreada indica los polígonos con $\geq 15$ pixeles en los cuadrantes de estudio (C1-C5)/

Shaded area indicates the polygons with $\geq 15$ pixels at the study quadrants (C1-C5).

\section{RESULTADOS Y DISCUSIÓN Conjunto total de datos}

Los resultados de las correlaciones se muestran en las Figuras 4-6. Utilizando el conjunto total de datos en $\mathrm{C1}-\mathrm{C} 5$, la amplitud de los valores de albedo resultó entre 15 y $55 \%$, y de temperatura entre 15 y $55^{\circ} \mathrm{C}$. De acuerdo con Dobos (2003), la amplitud de los valores de albedo corresponde a zonas de pastizal, zonas con suelo oscuro, suelo seco arcilloso, arena, suelo arcilloso seco o agua que coincide con las diferentes condiciones encontradas en C1-C5 (Figura 3).
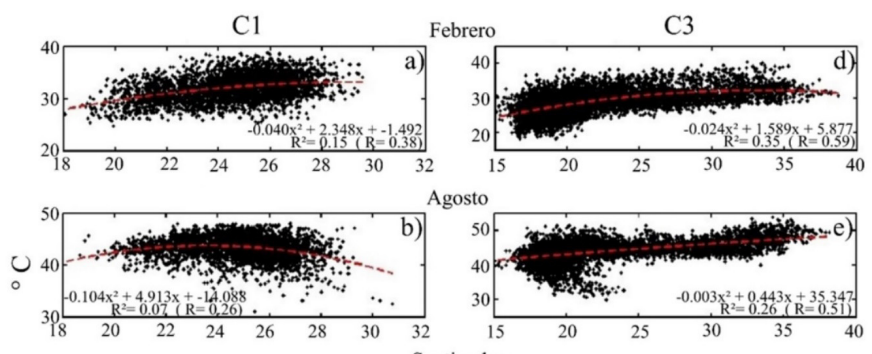

Agosto
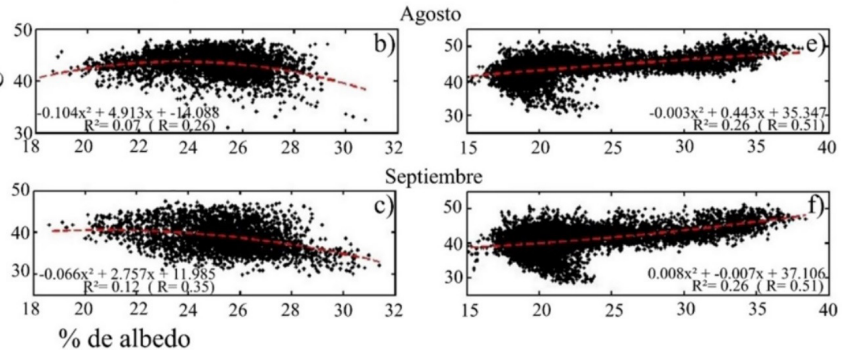

Figura 4. Correlación entre albedo y temperatura de todo el conjunto de datos, en los meses de febrero, agosto y septiembre para C1 (a, b, c) y C3 (d, e, f). C1 y C3 corresponden a los cuadrantes 1 y 3 .

Figure 4. Correlation between albedo and temperature using all records, in February, August and September for C1 (a, b, c) and C3 (d, e, f). C1 and C3 correspond to quadrants 1 and 3.

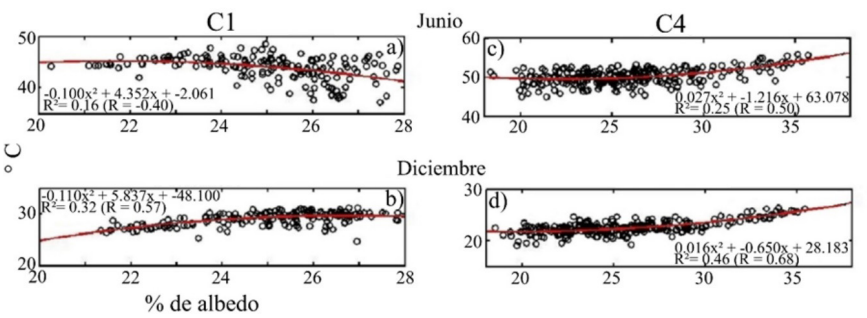

Figura 5. Correlación entre albedo y temperatura usando promedios históricos por mes de junio y diciembre en $\mathrm{C} 1(\mathrm{a}, \mathrm{b})$ y $\mathrm{C} 4(\mathrm{c}, \mathrm{d})$. C1 y C4 corresponden a los cuadrantes 1 y 4 .

Figure 5. Correlation between albedo and temperature using long-term averages per month for June and December in C1 (a, b) and C4 (c, d). C1 y C4 correspond to quadrants 1 and 4 .

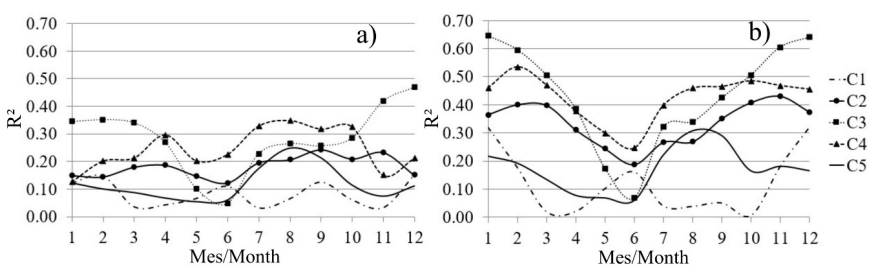

Figura 6. Comportamiento anual del coeficiente de determinación $\left(R^{2}\right)$ en los cuadrantes $\mathrm{C} 1-\mathrm{C} 5$, a) conjunto total de datos, b) promedio histórico mensual en los cuadrantes de estudio.

Figure 6. Annual behavior of the coefficient of determination $\left(R^{2}\right)$ in $C 1-C 5$ squares, a) all records, b) long-term averages per month. 
Los resultados del análisis de regresión entre albedo y temperatura superficial en cada uno de los cuadrantes fueron muy variados pero se encontraron correlaciones estadísticamente significativas en cada caso. La Figura 4, muestra las relaciones entre albedo y temperatura observados en C1 y C3 para los meses de febrero, agosto y septiembre. Las variaciones de $R^{2}$ en todos los meses y para C1-C5 de todo el conjunto de datos se muestran en la Figura 6a. En C1, por ejemplo, hubo valores de correlación, si bien modestos, que fueron estadísticamente significativos. El máximo coeficiente de determinación $\left(\mathrm{R}^{2}\right)$ en este caso, se presentó en los meses de enero, febrero y diciembre $(>0.12)$. Aquí solo se presentan los resultados del mes de febrero (Figura 4a), mientras que el mínimo en los meses de julio y noviembre (0.03). En los meses de invierno (noviembre-abril) la relación entre albedo y temperatura fue directa, es decir, conforme aumenta el albedo también se incrementa la temperatura. En los meses de verano (mayo-octubre), y principalmente en agosto (Figura 4b), la línea de regresión muestra un cambio en la pendiente para valores entre 22 y $24 \%$ de albedo indicando una transición de una relación directa anterior a una relación inversa posterior; en el mes de septiembre (Figura 4c), la relación entre albedo y temperatura se vuelve inversa para valores de albedo $\geq 24 \%$, es decir, conforme aumenta el albedo disminuye la temperatura. Este resultado es particularmente extraño puesto que septiembre es el mes más lluvioso de la región (Figura 2) resultando en incremento en la humedad del suelo y de la vegetación y, en teoría, se esperaría que la modulación de la temperatura estuviera dominada por el factor de evapotranspiración, debiendo resultar en una relación directa (Tereshchenko et al., 2012). De acuerdo con De Santa Olalla et al. (2005), si el contenido de agua en el suelo es suficiente, la evapotranspiración ocurrirá al máximo ritmo posible, pero existen otros factores que intervienen como el relieve y la extensión del territorio, ya que la distribución de lluvias puede no ser uniforme. En suelos oscuros y profundidades grandes, la radiación solar calienta las capas superiores, pero no todo el calor se emplea para la evapotranspiración, sino para calentar capas profundas produciendo almacenamiento de calor. Otra situación que debe tomarse en cuenta es que el tipo de vegetación que comprende esta área es efímera, en donde el reverdecimiento y floración de la vegetación es tan corto, que resultó poco perceptible entre las mediciones de un mes a otro.

Para el resto de los cuadrantes ( $C 2, C 3, C 4$ y C5), la relación entre albedo y temperatura resultó directa en todos los meses del año, indicando que el factor que modula la temperatura superficial es el de evapotranspiración. Particularmente en C3, la correlación entre albedo y temperatura fue mayor en febrero $(R=0.59)$, que en agosto y septiembre $(R=0.51)$. Al final de la temporada seca (mayo-junio) la correlación disminuye ( $R=0.32$ y 0.22 , respectivamente).

Con el conjunto total de datos (Figura 6a), en C1 los valores mínimos de $\mathrm{R}^{2}$ se presentaron en los meses de marzo, julio y noviembre. Por su parte en $\mathrm{C} 2$, los valores de $R^{2}$ se incrementan paulatinamente de febrero a abril y posteriormente disminuyen hasta el mes de junio. Después vuelven a incrementarse hasta alcanzar su máximo valor en septiembre.

En el cuadrante 3 , se observa un máximo de $\mathrm{R}^{2}$ en diciembre y un mínimo en junio. En el cuadrante 4 los valores de $R^{2}$, son máximos en abril, agosto y octubre, y mínimos en junio y en noviembre. Finalmente, en el cuadrante 5 , se observa un máximo de $\mathrm{R}^{2}$, en agosto y dos mínimos uno en junio y otro en noviembre.

\section{Promedios históricos por mes}

Los resultados del análisis de regresión entre el albedo y la temperatura superficial, utilizando los promedios históricos mensuales, al igual que en el caso del conjunto total de datos, las correlaciones encontradas fueron modestas, pero estadísticamente significativas en todos los cuadrantes. En la Figura 5, se muestran los resultados para $\mathrm{C} 1$ y $\mathrm{C} 4$, en los meses de junio (Figura $5 a$ y $5 c$ ) y diciembre (Figura 5 b y $5 d$ ).

La relación entre albedo y temperatura en $\mathrm{C} 1$, al final de la temporada seca (mayo-junio), principalmente en junio $\left(R^{2}=0.16\right)$ fue inversa (Figura $\left.5 a\right)$, indicando que las condiciones del paisaje en ese mes fueron lo suficientemente secas para que domine el factor de radiación en la modulación de la temperatura superficial. Durante los meses de noviembre a febrero (invierno) la relación cambió y se volvió directa, con $\mathrm{R}^{2}$ mayor en el mes de diciembre (Figura $5 \mathrm{~b}$ ); lo anterior indica que para estos meses el factor que dominó la modulación de la temperatura fue el de evapotranspiración.

Los resultados del análisis de regresión para C2-C5, mostraron que la relación entre albedo y temperatura fue directa en todos los meses.

Al considerar el promedio histórico mensual, las correlaciones entre albedo y temperatura aumentaron en muchos meses, pero mantuvieron en la mayoría de los casos los valores mínimos de $\mathrm{R}^{2}$ durante el mes de junio (Figura $6 \mathrm{~b})$, en $\mathrm{C} 1$, por ejemplo, los valores de $\mathrm{R}^{2}$ fueron los menores observados en relación a los valores de C2-C4. En este caso los valores mínimos de $\mathrm{R}^{2}$ se observaron en marzo y octubre. C2 mostró valores mínimos de correlación en el mes de junio, aumentando paulatinamente en los siguientes meses. C3 es el cuadrante que mostró cambios de mayor amplitud, iniciando con valores superiores a 0.60 en el mes de enero y descendió hasta el valor mínimo en junio $\left(R^{2}<0.10\right)$, mismo que siguió aumentando en los siguientes meses. C4 mantiene valores de $R^{2}$ superiores a 0.25 , con un máximo en febrero. C5 mostró un valor mínimo de $\mathrm{R}^{2}$ en el mes de junio y un máximo en el mes de agosto.

Se identificaron un total de 13 polígonos con diferentes tipos de vegetación y 15 polígonos con diferentes tipos de suelo. Para C1, el tipo de vegetación que cubre mayormente la superficie del cuadrante es matorral sarcocaule y sarcocrasicaule, con suelos de tipo Regosol; en C2 vegetación típica de desierto arenoso y suelos de tipo Regosol. En C3 la vegetación con mayor cobertura fue matorral sarcocaule y suelos de tipo Litosol; por su parte C4, mostró mayor vegetación de tipo matorral desértico y suelo tipo Regosol. En C5 se 
encontró vegetación típica de desierto arenoso y suelos de tipo Regosol calcárico.

Dentro de los polígonos con significancia estadística $(R \geq 0.50, a<0.05)$, el tipo de vegetación de matorral sarcocrasicaule en $\mathrm{C} 1$, así como de matorral desértico en C3 y C4, presentaron los cambios más relevantes en la relación albedo-temperatura a lo largo de los meses. El resto de los polígonos mantuvo una relación directa entre albedo y temperatura en todos los meses.

El matorral sarcocrasicaule mostró una relación inversa al final de la temporada seca, más evidente en el mes de junio (Figura 7a), que sugiere una dominancia del factor de radiación en la modulación de la temperatura superficial; en diciembre la correlación fue positiva, con un valor $\mathrm{R}=$ 0.69 (Figura 7b). Por su parte, el matorral desértico en C3, mostró en la temporada de verano correlaciones altas, con la característica que la línea de regresión mostró un cambio representado por una relación inversa para valores de albedo $<24 \%$ y directa, para valores de albedo $>28 \%$. Este cambio fue más notorio en el mes de mayo (Figura 7c); este resultado indica la presencia de parches de vegetación en los que se manifiesta la dominancia del factor de radiación y otros en los que, por el contrario domina el factor de evapotranspiración. De acuerdo con Granados-Sánchez et al. (1998), en las zonas áridas cada planta requiere de una superficie para proveerse de suficiente humedad y su distribución puede no ser uniforme. Además, analizando las condiciones secas del mes de mayo, posiblemente este sea un mes de transición donde se manifiestan ambas relaciones. En el mes de noviembre (Figura 7d), al iniciar la época de invierno, la correlación fue directa. Un comportamiento similar al observado en mayo,

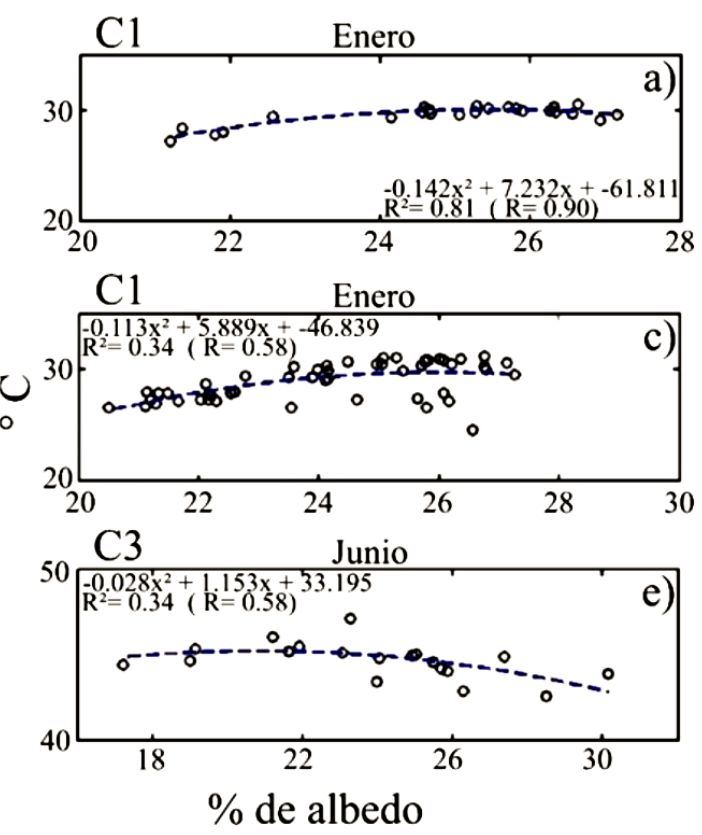

pero en este caso en C4 bajo el mismo tipo de vegetación, se observó en agosto (Figura 7e) y octubre (Figura 7f), aunque el valor de la correlación fue menor en ambos casos que el observado en C3. En los meses de invierno la relación fue directa, dominando el factor de evapotranspiración.

Cada planta posee, en cada estado particular de su desarrollo, una capacidad máxima de absorción que es función de las necesidades de sus células (Raven et al., 1992). Las estructuras que tienen un papel importante en la regulación del movimiento del agua en las plantas son los estomas; su apertura y cierre obran como compuertas de equilibrio. La iluminación, temperatura y humedad del aire, son factores que afectan este mecanismo. Ante esto es posible afirmar que, en la época de verano, al elevarse la temperatura, la vegetación no puede compensar la pérdida de humedad y comienza a entrar en estrés hídrico, expresando la dominancia del factor de radiación al final del verano.

Para los polígonos de tipo de suelo, con relaciones albedo vs. temperatura superficial estadísticamente significativas $(a<0.05)$, los suelos de tipo Regosol calcárico y Regosol éutrico (en C1), Yermosol háplico (en C2 y C3), y Vertisol crómico (en C3), fueron los que mostraron cambios en la pendiente de la línea de regresión a lo largo de los meses del año. La relación de los polígonos restantes se mantuvo con relaciones directas (indicando la dominancia del factor de evapotranspiración).

El tipo de suelo Regosol calcárico en C1 mostró durante el mes de enero una relación directa muy evidente $(R=$ 0.90) (Figura 8a); mientras que al final de la época seca, en el mes de junio, la relación cambia y pasó a ser inversa (Figura 8b). Este es un caso típico en el que el factor de evapotrans-
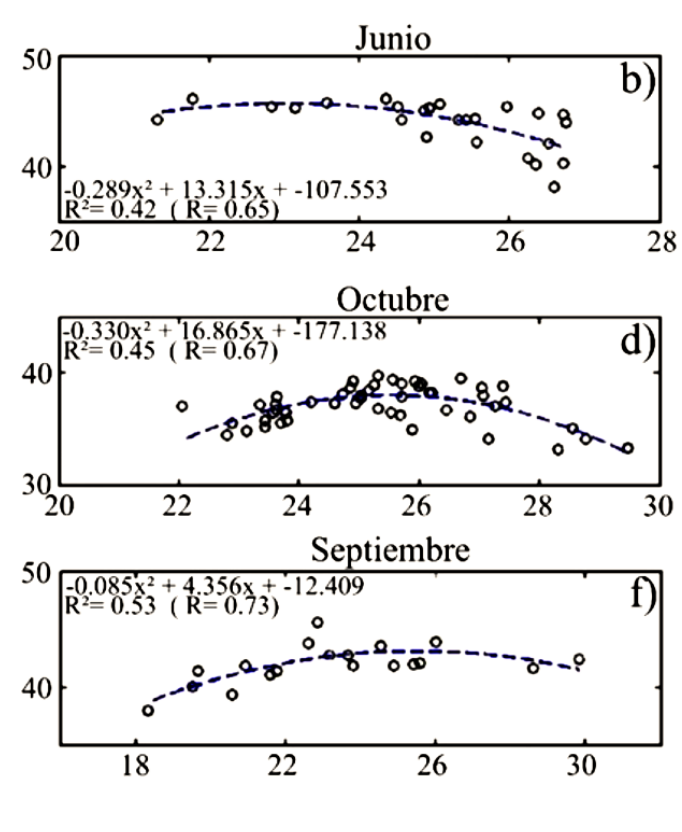

Figura 7. Correlaciones entre albedo y temperatura en áreas con diferente tipo de vegetación. De tipo matorral sarcocrasicaule en cuadrante 1 (C1) para (a) junio y (b) diciembre. Vegetación de tipo matorral desértico, en cuadrante 3 (C3) para (c) mayo y (d) noviembre; y para (e) agosto y (f) octubre, en cuadrante 4 (C4).

Figure 7. Correlations between albedo and temperature in areas with different vegetation types. Sarcocrasicaule scrub type at quadrant 1 (C1) for (a) June and (b) December. Desert scrub type, at quadrant 3 (C3) for (c) May and (d) November; at quadrant 4 (C4), (e) August and (f) October. 

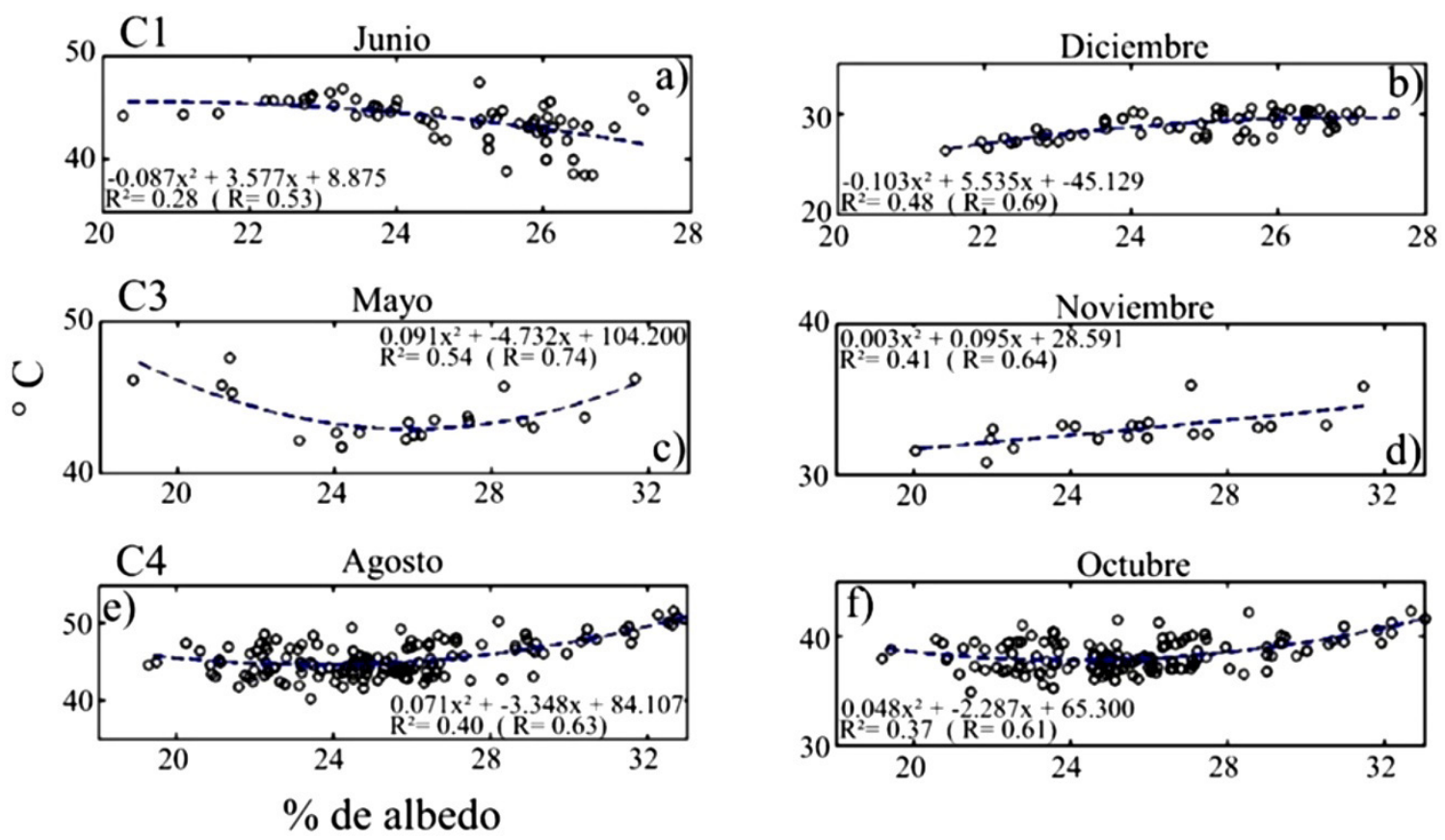

Figura 8. Correlaciones entre albedo y temperatura en diferentes tipos de suelo. Regosol calcárico en el cuadrante 1 (C1), para los meses de (a) enero y (b) junio; Regosol éutrico en el cuadrante 1 (C1), para los meses de (c) enero y (d) octubre; Vertisol crómico en el cuadrante 3 (C3), para los meses de (e) junio y $(\mathrm{f})$ septiembre.

Figure 8. Correlations between albedo and temperature in different soil type. Calcaric Regosol at quadrant 1 (C1), for (a) January and (b) June; Eutric Regosol at quadrant 1(C1), for (c) January and (d) October; Chromic Vertisol at quadrant 3 (C3), for (e) June and (f) September.

piración domina la modulación de la temperatura durante invierno, mientras que el factor de radiación domina al final de la época seca, cuando las plantas están mayormente deshidratadas. El tipo de suelo Regosol éutrico en el mismo cuadrante, mostró que en el mes de enero la relación entre albedo y temperatura fue directa (Figura 8c); mientras que en octubre, hay un cambio en la relación, pasando de ser directa para valores $\leq 25 \%$ de albedo e inversa para valores $>25 \%$ de albedo (Figura 8d).

Al tratarse de suelos tipo Yermosol háplico, la correlación entre albedo y temperatura fue mayor en diciembre y enero $(R=0.80)$, manteniendo una relación directa. Existieron cambios pocos perceptibles de transición durante los meses de verano. En C3, bajo condiciones de suelo Vertisol crómico, al inicio del verano (mayo y junio) se observó una relación inversa entre albedo y temperatura (Figura 8e), misma que fue cambiando en los siguientes meses a directa (septiembrefebrero) (Figura 8f).

De acuerdo a la Guía para la Interpretación de Cartografía Edafológica de INEGI (2014), las características de los tipos de suelo Regosol calcárico, Regosol éutrico, Yermosol háplico y Vertisol crómico, dan sustento a los tipos de relaciones encontradas, debido a que son suelos que permiten retener la suficiente cantidad de agua para que la vegetación establecida en ellos, pueda evapotranspirar en la temporada de invierno, pero que al incrementarse la temperatura en verano, disminuye la humedad en la capa superficial, llevando a la vegetación a un punto de estrés hídrico.

\section{CONCLUSIONES}

El análisis de las relaciones entre albedo y temperatura en diferentes sectores del Desierto Sonorense mostró cambios muy significativos asociados a las diferencias locales en cada cuadrante seleccionado. Cuando se analiza el conjunto total de datos en todos los pixeles de cada cuadrante, los resultados no son muy claros y, a veces hasta confusos, pues se observa una relación inversa entre albedo y temperatura durante los meses lluviosos, indicando que el factor que domina la modulación de temperatura es el de radiación, situación que contradice claramente a la teoría. Esto no es posible y una explicación a este resultado tiene que ver con el conjunto total de datos, en el que no se filtran los pixeles por tipo de suelo y de vegetación provocando una mezcla de condiciones que influyen en el análisis. Cuando se considera en el conjunto total de datos los promedios históricos mensuales, las correlaciones se incrementan un poco pero aun así no arrojan mucha luz a la identificación de los cambios en las relaciones. Por otro lado, cuando se realiza un filtro de los pixeles por los diferentes tipos de vegetación y de suelo, los resultados son mucho más favorables, llegando incluso a observarse el cambio en las relaciones entre albedo y temperatura en la dirección correcta, es decir, una relación directa en los meses de precipitación y una relación inversa durante la temporada seca, indicando que durante los meses de precipitación, cuando la vegetación es abundante domina el factor de evapotranspiración en la modulación de la temperatura, mientras que durante la temporada seca, cuando hay poca vegetación, el factor que domina la relación es el de 
radiación, indicando condiciones similares a las del desierto. Estos cambios se dan para valores de albedo entre 24 y $25 \%$. Sin embargo, los resultados no son consistentes en todos los cuadrantes ni en todas las épocas, aun así, arrojan un poco de luz para estudios de sequía, pues los valores de albedo indicadores del cambio, pueden ayudar a entender mejor las temporadas en que inicia el período seco que eventualmente se puede convertir en una sequía.

\section{AGRADECIMIENTOS}

El financiamiento de esta investigación lo otorgaron los proyectos PC 0.3 de CIBNOR y REDESClim de CONACYT (Registro 254533). Los autores agradecen el apoyo del Ing. Julio Félix Domínguez, el M. en C. Edgar Alcántara Razo y la Dra. Tatiana Titkova, por su apoyo en la obtención de datos y procesamiento de información satelital. MGGM es becaria del Consejo Nacional de Ciencia y Tecnología (CONACYT No. Becaria 300458).

\section{REFERENCIAS}

Charney, J. G. 1975. Dynamics of deserts and drought in the Sahel. Quarterly Journal of the Royal Meteorological Society. Vol. 101(193-202).

De Santa Olalla, F. M., López, F. P. y Calera, B. A. 2005. Agua y Agronomía. Ed. Mundi-Prensa. 166-173.

Dobos, E. 2003. Albedo. Encyclopedia of Soil Science. DOI: 10.1081/E-ESS 120014334

Douglas, M. W., Maddox, R. A., Howard, K. y Reyes, S. 1993. The Mexican Monsoon. Journal of Climate 6:1665-1677.

Granados-Sánchez, D., López-Ríos, G. y Gama-Flores, J. 1998. Adaptaciones y estrategias de las plantas de Zonas Áridas. Rev. Chapingo. Ciencias Forestales del Ambiente 4(1): 169178.

Higgins, R. W., Douglas, A. V., Hahmann, A., Berbey, E. H., Gutzler, D., Shuttleworth, J., Stensrud, D., Amador, J., Carbone, R., Cortez, M., Douglas, M. W., Lobato, R., Meitin, J., Ropelewski, Ch., Schemm, J., Schubert, S. y Zhang, Ch. 2003. Progress in Pan American CLIVAR research: the North American monsoon system. Atmósfera 16(1): 29-65.

INEGI. 2013a. Regiones naturales y biogeográficas de México. INEGI, Dirección de capacitación. 19-22.
INEGI. 2013b. Carta de Uso de Suelo y Vegetación. Serie continua V. 1:250,000. [Consultado 6 Noviembre 2017]. Disponible en: http://www.beta.inegi.org.mx/app/mapas/

INEGI. 2014. Guía para la Interpretación de Cartografía: Edafología. Escala 1:250 000. Serie III. Instituto Nacional de Estadística y Geografía. México. 69 p.

INIFAP-CONABIO. 1995. Mapa edafológico. Escala 1:250000 y 1:1000000. Instituto Nacional de Investigaciones Forestales y Agropecuarias/Comisión Nacional para el Conocimiento y Uso de la Biodiversidad. México.

Jáuregui, E. 1995. Rainfall fluctuations and tropical storm activity in Mexico. Erkunde (49): 39-48.

Otterman, J. 1974. Baring high-albedo soils by overgrazing: Hypothesized desertification mechanism. Science, 186(4163): 531-533.

Pallé, E., Goode, P. y Montañés-Rodríguez, P. 2008. Inter-annual variations in Earth's reflectance 1999-2007. Journal of Geophysical Research. 1-21.

Raven, P., Evert, R. y Eichhorn, S. 1992. Biología de las Plantas. Ed. Reverté. 548-551.

Schaaf, C. y Wang, Z. 2015. MCD43C3 MODIS/Terra+Aqua BRDF/ Albedo Albedo Daily L3 Global 0.05 Deg CMG V006 [Data set]. NASA EOSDIS Land Processes DAAC.

Shreve, F. y Wiggins, I. 1964. Vegetation and Flora of the Sonoran Desert. Stanford University Press, $1740 \mathrm{p}$.

Taylor, F. W. 2005. Elementary Climate Physics. Oxford University Press, U.K.

Tereshchenko, I., Zolotokrylin, A., Titkova, T. B., Brito-Castillo, L. y Monzón, C. O. 2012. Seasonal variation of surface temperature-modulating factors in the Sonoran Desert in Northwestern Mexico. Journal of Applied Meteorology and Climatology, 51: 1519-1530 p.

Vivoni, E., Moreno, H., Mascaro, G., Rodríguez, J., Watts, C., Garatuza-Payan., J. y Scott, R. 2008. Observed relation between evapotranspiration and soil moisture in the North American monsoon region. Geophysical Research Letters, 35, L22403.

Wan, Z., Hook, S. y Hulley, G. 2015. MOD11C3 MODIS/Terra Land Surface Temperature/Emissivity Monthly L3 Global 0.05 Deg CMG V006 [Data set]. NASA EOSDIS LP DAAC.

Zolotokrylin, A. 2003. Climate desertification. Nauka, Moscow. 